\title{
THE METHOD OF GENERALIZED RAY-REVISITED
}

\author{
Franz Ziegler * $\quad$ Piotr Borejko ${ }^{* *}$ \\ Department of Civil Engineering \\ Technical University of Vienna \\ Vienna, A-1040, Austria-Europe
}

In Section 2, ROTATION OF COORDINATES, the Authors derived the emittance functions in the WeylSommerfeld representation of the wave potentials for a horizontal instantaneous single force from those known for a vertical force from conditions of invariance of the phase and amplitude of plane waves under coordinate rotation, Eqs. $(10) \sim(13)$ and $(18) \sim(20)$. That transformation implies the validity of the commonly applied identity for the (force) vector components when rotating the vector in the opposite sense to the coordinate rotation. Further, in the three-dimensional case, the vertical force poses an axisymmetric problem which is compatible with the Fourier transformation applied to the coordinates in the horizontal plane. When rotating the coordinates (or the force) by 90 degrees, the problem is physically still axisymmetric, however, the choice of the independent wave slowness in the horizontal plane no longer allows the invariant combination of the squared sum of a horizontal and a vertical wave slowness, the latter is different for $\mathrm{P}$ - and S-waves. Therefore, and because of the "additional" assumption that after rotation, one component of the vectorial potential of the S-waves still vanishes, the results of the emittance functions Eqs. (13), (15), (20), (23) and the superposition, Eqs. (25) (28) are wrong for three-dimensional waves. Putting one horizontal wave slowness to zero in these expressions, renders the cylindrical waves produced by vertical or horizontal line sources, and for the two-dimensional problem the results derived by the coordinate rotation remain valid. Hence, for the horizontal force in the $x$-direction, the following boundary value problem in the Laplace and Fourier transformed stresses Eq. (3) and displacements Eq. (1) must be solved:

$z=0: \widetilde{\bar{\sigma}}_{z x}(\xi, \kappa, s)=-\frac{1}{2} \varepsilon_{z} Q_{x} \bar{f}(s), u_{z}=u_{y}=0$
The 4th equation is given by Eq. $\left(1_{2}\right)$. The force in the horizontal $y$-direction requires the solution of

$z=0: \widetilde{\bar{\sigma}}_{z y}(\xi, \kappa, s)=-\frac{1}{2} \varepsilon_{z} Q_{y} \bar{f}(s), u_{z}=u_{x}=0$

In both cases the emittance functions exhibit a strong coupling. However, a rotation about the vertical $z$-axis through 90 degrees (with counter-rotation of the horizontal force) reproduces the results as given by the solutions of the boundary value problems. The source functions in Table 1 have been derived by means of the symbolic manipulation program Mathematica. Putting either the slowness $i \kappa$ or the slowness $i \xi$ to zero yields the emittance functions of the horizontal line loads in the $x$ - or $y$-directions, respectively. The following determinant of the respective system of four linear equations (only Eqs. (C1) or (C2) are non-homogeneous) is used, respectively,

$$
\Delta_{x}=\eta\left(\zeta^{2}-\xi^{2}\right)-\zeta \kappa^{2}, \quad \Delta_{y}=\eta\left(\zeta^{2}-\kappa^{2}\right)-\zeta \xi^{2}
$$

Table 1

\begin{tabular}{|l|l|}
\hline Substitute in Eq. (13) & Substitute in Eq. (20) \\
\hline$S_{P}^{x}=C^{-2} \frac{i \xi}{\eta} \frac{\eta}{\Delta_{x}}$ & $S_{P}^{y}=C^{-2} \frac{i \kappa}{\eta} \frac{\eta}{\Delta_{y}}$ \\
$S_{S x}^{x}=\varepsilon_{z} \xi \kappa \frac{\eta-\zeta}{\Delta_{x}}$ & $S_{S x}^{y}=-\varepsilon_{z}$ \\
$S_{S y}^{x}=\varepsilon_{z}$ & $S_{S y}^{y}=-\varepsilon_{z} \xi \kappa \frac{\eta-\zeta}{\Delta_{y}}$ \\
$S_{S z}^{x}=i \kappa \frac{\eta \zeta-\xi^{2}-\kappa^{2}}{\Delta_{x}}$ & $S_{S z}^{y}=-i \xi \frac{\eta \zeta-\xi^{2}-\kappa^{2}}{\Delta_{y}}$ \\
\hline
\end{tabular}

* Professor, Fellow ASME \& IIAV ** Docent in Seismology 
Substitute for Eq. (15) the non-vanishing Weyl-Sommerfeld integral

$\bar{\psi}_{x}^{x}(x, y, z, s)=s^{2} Q_{x} \bar{F}(s) \int_{-\infty}^{\infty} \int_{-\infty}^{\infty} S_{S x}^{x} \exp \left(s g_{S}\right) d \xi d \kappa$

and for Eq. (23)

$\bar{\psi}_{y}^{y}(x, y, z, s)=s^{2} Q_{y} \bar{F}(s) \int_{-\infty}^{\infty} \int_{-\infty}^{\infty} S_{S y}^{y} \exp \left(s g_{S}\right) d \xi d \kappa$

to find the correct superposed emittance functions, the corrected Eqs. (25) (28),

$$
\begin{aligned}
S_{P} & =Q_{x} S_{P}^{x}+Q_{y} S_{P}^{y}+Q_{z} S_{P}^{z} \\
& =Q_{x} \frac{i \xi C^{-2}}{\Delta_{x}}-Q_{y} \frac{i \kappa C^{-2}}{\Delta_{y}}-Q_{z} \varepsilon_{z}
\end{aligned}
$$

$$
\begin{aligned}
S_{S x} & =Q_{x} S_{S x}^{x}+Q_{y} S_{S x}^{y}+Q_{z} S_{S x}^{z} \\
& =Q_{x} \frac{\varepsilon_{z} \xi \kappa(\eta-\zeta)}{\Delta_{x}}-Q_{y} \varepsilon_{z}-Q_{z} \frac{i \kappa}{\zeta}
\end{aligned}
$$

$$
S_{S y}=Q_{x} S_{S y}^{x}+Q_{y} S_{S y}^{y}+Q_{z} S_{S y}^{z}
$$

$$
=Q_{x} \varepsilon_{z}-Q_{y} \frac{\varepsilon_{z} \xi \kappa(\eta-\zeta)}{\Delta_{y}}+Q_{z} \frac{i \xi}{\zeta}
$$

$S_{S z}=Q_{x} S_{S z}^{x}+Q_{y} S_{S z}^{y}+Q_{z} S_{S z}^{z}$

$$
=\left[Q_{x} \frac{i \kappa}{\Delta_{x}}-Q_{y} \frac{i \xi}{\Delta_{y}}\right]\left(\eta \zeta-\xi^{2}-\kappa^{2}\right)
$$

The rotations of coordinates as indicated in the Figs. 1 and 2 and the invariance conditions of phase and amplitude should be applied only for the sake of representing one and the same set of three-dimensional potential functions in two reference systems. 